\title{
BMJ Open Effect of preoperative duloxetine treatment on postoperative chronic residual pain after total hip or knee arthroplasty: a randomised controlled trial
}

Wietske Rienstra (D) , ${ }^{1,2}$ Tim Blikman (D) , ${ }^{1,2}$ Baukje Dijkstra, ${ }^{3}$ Roy Stewart (D) ,,5 Wierd Zijlstra, ${ }^{3}$ Tom van Raaij, ${ }^{6}$ Anita ten Hagen, ${ }^{7}$ Sjoerd Bulstra, ${ }^{2}$ Martin Stevens (D) , ${ }^{2}$ Inge van den Akker-Scheek (1) ${ }^{2}$

To cite: Rienstra W, Blikman T, Dijkstra B, et al. Effect of preoperative duloxetine treatment on postoperative chronic residual pain after total hip or knee arthroplasty: a randomised controlled trial. BMJ Open 2021;11:e052944. doi:10.1136/ bmjopen-2021-052944

- Prepublication history and additional supplemental material for this paper are available online. To view these files, please visit the journal online (http://dx.doi.org/10.1136/ bmjopen-2021-052944).

Received 02 May 2021 Accepted 18 0ctober 2021
Check for updates

(C) Author(s) (or their employer(s)) 2021. Re-use permitted under CC BY-NC. No commercial re-use. See rights and permissions. Published by BMJ.

For numbered affiliations see end of article.

Correspondence to

Dr Inge van den Akker-Scheek; i.scheek@umcg.nl

\section{ABSTRACT}

Objectives A key predictor for developing chronic residual pain after total knee or hip arthroplasty (TKA/ THA) is sensitisation. Sensitisation can be defined as an 'increased responsiveness of nociceptive neurons in the nervous system'. Aim of this study is to investigate the effects of preoperative treatment with duloxetine in sensitised knee and hip osteoarthritis (OA) patients on postoperative chronic residual pain up to 1 year after arthroplasty.

Setting A multicentre, pragmatic, prospective, randomised clinical trial was conducted in three secondary care hospitals in the Netherlands.

Participants Patients with primary knee/hip OA who were planned for TKA/THA were screened using the modified painDETECT Questionnaire. Patients whose painDETECT score indicated that sensitisation may be present were eligible for participation. 111 participants were included and randomly assigned 1:1 to an intervention or control group. The intervention group received additional duloxetine treatment, the control group did not receive any additional treatment but was allowed to continue with any pain medication they were already taking. Interventions Preoperative oral treatment for 7 weeks with $60 \mathrm{mg} /$ day of duloxetine was compared with usual care.

Primary and secondary outcome measures Primary outcome measure was pain at 6 months after arthroplasty, assessed with the Pain Subscale of the Knee injury and Osteoarthritis Outcome Score (KOOS) or the Hip disability and Osteoarthritis Outcome Score (H0OS) with a 0-100 scale. Secondary outcome measures were Visual Analogue Scale (VAS), and neuropathic-like pain measured using the modified PainDETECT Questionnaire. Longitudinal data collection included time points directly after duloxetine treatment, 1-day preoperatively, and 6 weeks, 6 months and 12 months postoperatively.

Results Mean improvement in the KOOS/HOOS pain subscale at 6 months postoperatively was 37 (SD 28.1) in the intervention group and 43 (SD 26.5) in the control group. No statistically significant difference was found in change score 6 months postoperatively between the two

\section{STRENGTHS AND LIMITATIONS OF THIS STUDY}

$\Rightarrow$ Broad screening of all patients who were planned for total knee or hip arthroplasty, creating a representative study population.

$\Rightarrow$ Using patient-reported outcome measures relevant for clinical practice.

$\Rightarrow$ Comparing to usual care, which varied among clinicians and participating centres, thereby increasing generalisability.

$\Rightarrow$ Long-term follow-up focusing on clinical relevance of the efficacy of duloxetine treatment from prior to arthroplasty to postoperative outcome.

$\Rightarrow$ The substantial difference in treatment effect of duloxetine between hip and knee osteoarthritis patients was not anticipated and somewhat lessens the interpretability of our results for the total study group.

groups $(p=0.280) .12$ patients from the intervention group (21\%) discontinued duloxetine due to adverse effects. Conclusions Preoperative targeted treatment with duloxetine in end-stage knee and hip OA patients with sensitisation does not influence postoperative chronic residual pain after TKA/THA.

Trial registration number NTR4744.

\section{INTRODUCTION}

Total hip and knee arthroplasties are among the most performed procedures in orthopaedic surgery for the treatment of patients with severe osteoarthritis (OA). ${ }^{12}$ Projections show that the number of performed procedures will dramatically rise in the future. ${ }^{3-5} \mathrm{In}$ light of this, the high prevalence of residual pain after these procedures must be considered a highly relevant problem. Chronic residual pain is pain that persists after the postoperative recovery process is over. Up to $23 \%$ of patients after total hip arthroplasty 
(THA) and up to $34 \%$ after total knee arthroplasty (TKA) experience chronic residual pain, ${ }^{6-10}$ which leads to declining patient satisfaction, functioning and quality of life. ${ }^{11-14}$

Numerous studies have demonstrated that pain in OA is a highly complex phenomenon that seems to involve both intra-articular and extra-articular mechanisms ${ }^{1713-16}$ like modification of pain transmission in both the peripheral and the central nervous system, leading to sensitisation of the pain pathways. Several mechanisms have been described leading to sensitisation, among which modulation of the inhibitory descending control pathways of the central nervous system seems to play an important role. $^{717}$ Sensitisation in OA expresses itself through neuropathic-like symptoms such as allodynia, hyperalgesia and spreading of the pain. Signs of sensitisation seem to be among the key predictors for poorer outcome after total joint arthroplasty (TJA), especially for chronic residual pain. ${ }^{18-22} \mathrm{Up}$ to $19 \%$ of patients with hip OA and $19 \%-37 \%$ of patients with knee OA experience signs of sensitisation and are therefore at a higher risk of developing chronic residual pain after TJA. ${ }^{710-14} 1623$

As sensitisation in $\mathrm{OA}$ is an important risk factor for developing chronic residual pain after THA/TKA, it is plausible that targeted treatment aimed at preoperative desensitisation, for example, with neuromodulating medication, will reduce chronic residual pain. Duloxetine, a selective serotonin and norepinephrine reuptake inhibitor, influences the descending inhibitory control pathways of the central nervous system. A recent metaanalysis shows that duloxetine has a positive effect on pain in OA patients. ${ }^{24-27}$ A recent study shows that use of duloxetine perioperatively ( 1 day before up to 6 weeks after surgery) in sensitised knee OA patients has positive effects on pain up to 12 weeks postoperatively. ${ }^{27}$ To our knowledge, it is unknown whether this beneficial effect remains in long-term follow-up. Specifically selecting OA patients with signs of sensitisation rather than the general knee and hip OA population will enable a better assessment of the effectiveness of pre-THA/TKA desensitisation on the development of chronic residual pain. Until now, the effect of duloxetine on pain in OA patients has solely been investigated compared with placebo. It is of clinically relevant value to assess the added effect of duloxetine in OA patients compared with usual care.

Aim of this study is, therefore, to investigate whether preoperative treatment with duloxetine of hip and knee OA patients with signs of sensitisation reduces postoperative chronic residual pain up to 1 year post-TJA.

\section{METHODS}

\section{Design}

This article describes the outcome of a multicentre, pragmatic, prospective, open-label, randomised clinical tria. Participating hospitals were University Medical Center Groningen (UMCG), Martini Hospital Groningen and Medical Center Leeuwarden. A detailed description of the study design was published earlier. ${ }^{28}$ No important changes were made to the methods and no changes were made to trial outcomes after commencement of the trial. Authors TB and WR generated the random allocation sequence, enrolled participants and assigned participants to interventions.

The procedures followed were in accordance with the ethical standards of the responsible committee on human experimentation and with the Declaration of Helsinki of 1975, as revised in 2000.

\section{Patient and public involvement}

Neither patients nor the public were involved in the design, conduct, reporting or dissemination plans of our research.

\section{Participants and screening}

Patients were recruited between December 2014 and June 2018; follow-up was completed in 2019. During the study period, all patients with primary hip or knee OA planned for THA or TKA were screened using a self-report tool for neuropathic-like pain symptoms in hip and knee OA, the modified PainDETECT Questionnaire (m-PDQ). The $\mathrm{m}-\mathrm{PDQ}$ is a self-administered questionnaire consisting of 12 items on neuropathic pain symptoms in the left/ right knee or hip during the past week. The questions ask about presence of pain radiation; pattern of pain over time; pain quality, including burning, tingling or prickling sensation; pain at light touch; sudden pain attacks; pain at cold or warm stimulus; numbness and pain at light pressure. The total score ranges from -1 to 38 points. Analogously to the original PDQ, scoring $\leq 12$ indicates a nociceptive pain profile, 13-18 a possible neuropathic pain profile and $\geq 19$ a likely neuropathic pain profile. Apart from nociceptive discrimination from neuropathic pain, m-PDQ scores $>12.0$ are associated with greater odds of having signs of sensitisation. ${ }^{89-31}$ Online supplemental file includes more detailed information on the m-PDQ. Patients who reported m-PDQ scores $>12.0$ and were eligible based on the inclusion and exclusion criteria were invited to participate.

Exclusion criteria were: surgical hip or knee joint procedures in the past year; intra-articular hip/knee injection/ arthroscopy in the past 3 months; cognitive or neurological disorders that could strongly interfere with questionnaire surveys; a history of significant peripheral nerve injury; serious or unstable physical or mental medical conditions that could interfere with study participation; intended THA/TKA to another joint within the study period; previous exposure to duloxetine or a medical contraindication for usage of duloxetine. A complete list of inclusion and exclusion criteria can be found in the design paper. ${ }^{28}$

Patients received oral and written information plus 2 weeks' consideration time. Patients willing to participate were invited to visit the outpatient clinic of their orthopaedic department, where the last safety-related exclusion criteria were ruled out based on laboratory 
testing and physical examination. Patients who complied with the inclusion and exclusion criteria and were still willing to participate, provided written informed consent and their visit to the outpatient clinic extended into a baseline visit.

\section{Randomisation}

Randomisation took place with a 1:1 allocation ratio. The ALEA online randomisation programme (ALEA, FormsVision, Abcoude, The Netherlands) localised on the secured servers of the local Trial Coordination Centre of UMCG was used. Participants were stratified by type of arthroplasty to be performed (hip or knee), with block sizes of 4 and 6.

\section{Procedure}

Demographic information, patient characteristics and medical history were collected using patient records (see table 1), and all patients received their first set of questionnaires at baseline. The preoperative treatment period was divided into three time points. As the risk of side effects was highest at initiation of treatment, the dosage of duloxetine was built up from $30 \mathrm{mg} /$ day in week 1 to $60 \mathrm{mg} /$ day in week 2 . The first time point, 2 weeks after baseline, was therefore primarily instated for safety reasons and to assess side effects. The second time point was 8 weeks after baseline, right after the 7-week treatment period with $60 \mathrm{mg}$ /day duloxetine. This time point aimed

Table 1 Demographics and baseline characteristics

\begin{tabular}{|c|c|c|c|c|}
\hline Characteristics & Total (111) & Duloxetine (57) & Care as usual (54) & $P$ value \\
\hline Age & $62.7(8.5)$ & $61.5(8.1)$ & $64.0(8.7)$ & 0.114 \\
\hline Gender (female) & $69(62.2)$ & $38(66.7)$ & $31(57.4)$ & 0.334 \\
\hline Cohabitation $(n=110)$ & $84(76.4)$ & $43(76.8)$ & $41(75.9)$ & 0.999 \\
\hline Education & & & & 0.768 \\
\hline Higher & $44(39.6)$ & $23(40.4)$ & $21(38.9)$ & \\
\hline Secondary & $59(53.2)$ & $29(50.9)$ & $30(55.6)$ & \\
\hline None or lower & $8(7.2)$ & $5(8.8)$ & $3(5.6)$ & \\
\hline BMI & $28.9(4.5)$ & $28.8(5.0)$ & $29.0(3.9)$ & 0.874 \\
\hline Smoking & $21(18.9)$ & $15(26.3)$ & $6(11.1)$ & 0.053 \\
\hline Knee & $61(54.9)$ & $31(54.4)$ & $30(55.6)$ & 0.999 \\
\hline Duration of pain (months) & $42.0(18 ; 7)$ & $48(22.5 ; 90)$ & $36(16 ; 66.8)$ & 0.312 \\
\hline Past surgery in index joint & 59 (53.2) & $30(52.6)$ & $29(53.7)$ & 0.999 \\
\hline ASA score $(n=110)$ & & & & 0.169 \\
\hline I & $34(30.9)$ & 19 (33.9) & $15(27.8)$ & \\
\hline II & 67 (60.9) & $31(54.4)$ & $37(68.5)$ & \\
\hline III & $9(8.2)$ & $7(12.5)$ & $2(3.7)$ & \\
\hline $\mathrm{KL}$ grade & & & & 0.167 \\
\hline II & $23(20.7)$ & $8(14.0)$ & $15(27.8)$ & \\
\hline III & $82(73.9)$ & 45 (78.9) & $37(68.5)$ & \\
\hline IV & $6(5.4)$ & $4(7.0)$ & $2(3.7)$ & \\
\hline \multicolumn{5}{|l|}{ KOOS/HOOS (0-100) } \\
\hline Pain & $34.4(13.8)$ & $38.1(14.0)$ & $30.6(12.7)$ & 0.004 \\
\hline Symptoms & $42.3(16.8)$ & $43.4(18.7)$ & $41.1(14.6)$ & 0.471 \\
\hline ADL & $40.2(14.9)$ & $41.7(15.2)$ & $38.6(14.6)$ & 0.270 \\
\hline QOL & $23.5(13.4)$ & $25.4(13.8)$ & $21.4(12.8)$ & 0.114 \\
\hline mPDQ (-1-38) & $15.8(4.6)$ & $15.6(4.7)$ & $16.0(4.6)$ & 0.659 \\
\hline VAS-R (110) & $52.6(22.6)$ & $46.6(24.8)$ & $58.7(18.2)$ & 0.004 \\
\hline VAS-M (111) & $69.5(16.4)$ & $68.1(15.6)$ & $71.1(17.2)$ & 0.337 \\
\hline
\end{tabular}

Dichotomous/categorical N (\%), $\chi^{2}$ test. Continuous, normally distributed mean (SD), Student's t-test (normality tested by histogram). Continuous, not normally distributed median (Q1; Q3), Mann-Whitney U test.

ADL, activities of daily living; ASA score, American Society of Anesthesiologists score; BMI, body mass index; KL grade, Kelgren and Lawrence grade; KOOS/HOOS, Knee injury and Osteoarthritis Outcome Score/Hip disability and Osteoarthritis Outcome Score; mPDQ, modified painDETECT Questionnaire; QOL, quality of life; VAS-M, Visual Analogue Scale for pain during Movement; VAS-R, VAS for pain in Rest. 
to measure the effect of duloxetine on pain directly after treatment. Before surgery the dosage of duloxetine was tapered for 2 weeks to $30 \mathrm{mg} /$ day, to reduce discontinuation symptoms. For safety reasons related to possible influence of duloxetine on platelet function, there was a window of 5-8 days between ending the duloxetine treatment period and surgery. The third and last preoperative time point took place 1 day prior to surgery. Patients in the care-as-usual group were mailed identical sets of questionnaires at the same time points.

Surgery and the postoperative recovery process followed local protocols. No study-related measures were needed. All participants of the two study groups were mailed identical sets of questionnaires at 48 hours, 6 weeks, 6 months and 12 months postoperatively, to assess the effect of the duloxetine treatment on the endpoints at different follow-up stages. A detailed description of all measurement instruments used and the time points at which they were administered can be found in the design paper and in the online supplemental file. ${ }^{28}$

\section{Intervention}

Patients randomised for the intervention group received duloxetine added to their usual care for 10 weeks preoperatively. The recommended dosage for chronic musculoskeletal pain is $60 \mathrm{mg} /$ day when considering maximal effectiveness and minimal side effects. ${ }^{32}$ Based on previous studies a 7-week treatment period with $60 \mathrm{mg}$ / day was considered sufficient to establish a relevant effect on pain. ${ }^{334}$ The total intervention period was 10 weeks, including 1 week of build-up and 2 weeks of tapering off the medication dose as described above. See online supplemental file for a visual illustration of the intervention phase.

\section{Usual care}

Patients in the usual-care group remained on the waiting list for arthroplasty. They were allowed to continue with any pain medication they were already taking as well as any other ongoing treatment (like physiotherapy). Since the use of neuropathic pain medication is not registered for OA pain in the Netherlands, none of the participants had a prescription for such medication in the preoperative stage. Usual care patients received regular preoperative care following local protocols, without imposed procedures. No restrictions were imposed on usage of escape pain medication in either study group-with one exception, that of agents specifically targeted for neuropathic pain, like gabapentinoids perioperatively.

\section{Measurement instruments}

Primary endpoint is the mean difference between the intervention and control groups in hip-specific or kneespecific pain 6 months postoperatively, assessed with the pain subscale of the Knee injury and Osteoarthritis Outcome Score (KOOS) or the Hip disability and Osteoarthritis Outcome Score (HOOS). Both KOOS and HOOS use a $0-100$ scale, where 0 represents extreme symptoms and 100 no symptoms. In literature a score $<70$ points on the KOOS or HOOS pain subscale is considered a moderate amount of joint-specific pain. ${ }^{35}{ }^{36}$ Missing items in the KOOS/HOOS were handled according to the KOOS/HOOS manual. ${ }^{35} 36$ This primary outcome measure was chosen at 6 months, as in practice this was considered the first possible time point to evaluate chronic residual pain after arthroplasty. Because it is known from practice that the amount of chronic residual pain is not likely to change after 1 year postop, we aimed to follow up to 1-year postoperative in order to be as thorough as possible.

Secondary study endpoints included:

1. The effect of treatment on change in general pain 6 months postoperatively, measured using a VAS ( $100 \mathrm{~mm}$ horizontal line representing pain from 0 (no pain) to 100 (worst pain imaginable)).

2. Amount of neuropathic-like pain measured using the modified PainDETECT-Questionnaire (m-PDQ) 6 months postoperatively.

3. Course of the above-mentioned pain scores at different time points. A detailed description of all time points and the measurements performed during those time points is provided in the design paper. ${ }^{28}$ Time points 1, 2 and 3 cover the preoperative intervention phase, time points 4, 5, 6 and 7 cover the postoperative period, ranging from 48 hours (the primary outcome measure was not assessed at this time point) to 6 weeks, 6 months and 12 months, respectively. See also online supplemental figure 1 for a visual overview of the study time points. As time points 1, 3 and 4 were appointed primarily for the evaluation of side effects, discontinuation effects or perioperative complications and not for the evaluation of the primary outcome measures, these time points were omitted from analyses in the present paper.

\section{Sample size calculation}

Sample size calculation was based on the primary endpoint: difference in the KOOS/HOOS pain subscale at 6 months after arthroplasty between the intervention and control group. According to literature, the preoperative mean (SD) pain subscale scores for the KOOS and HOOS are 35.9 (17.2) and 32.7 (17.7), respectively, and the minimally clinical important difference is 10 points. ${ }^{37}$ To detect a difference with $80 \%$ power $(0.05$ two-sided significance level), a total sample size of 47 participants per group was needed (94 participants in total). To account for an estimated $20 \%$ rate of protocol violators/ dropouts we aimed to include 118 participants.

\section{Statistical analysis and handling of data}

Statistical analyses were performed using IBM SPSS Statistics for Windows (V.22.0, IBM). Descriptive statistics were used to report patient characteristics, using mean and SD or median and percentiles in case of continuous variables, based on normality assessment by histogram. For normally distributed data, differences between treatment 
groups were assessed using an independent samples Student's t-test. For non-normally distributed data a MannWhitney U-test was performed. Our planned analysis was the inferential test between difference in KOOS/HOOS pain subscale between the intervention and the control group at 6 months after surgery. Proportions and percentages were described for discrete data, In case of discontinued participation in the study, all data obtained up to the participant's discontinuation was analysed according to the intention-to-treat principle. All participants with at least one measurement after baseline were included in the study analyses. The data were not imputed. We decided to use a Full Information Maximum Likelyhood technique using multilevel mixed model analysis for repeated measures. Multilevel models have the ability to handle models by using all available data, which is an advance over traditional repeated-measures analysis, where the usual treatment is to remove the entire patient if one of the outcomes is missing. With the multilevel model, we use as estimated strategy full information maximum likelihood, where we get parameter estimates even in the presence of missing data. Missing items in the primary outcome scores, the pain subscales of the KOOS/HOOS questionnaires, were handled according to the KOOS/ HOOS manual. ${ }^{3536}$

A multilevel mixed model analysis for repeated measures was performed on the longitudinal data to determine whether there is a difference in the modification of pain over time between the two groups. A mixed model was constructed that included time, treatment allocation and baseline KOOS/HOOS pain subscale (in order to correct for the differences between groups at baseline). A variable was added differentiating between preoperative and postoperative time points (coded 0 or 1 , respectively), thereby creating a piece-wise analysis. This way the postoperative effect of duloxetine treatment could be distinguished while including the data from all time points. Apart from the baseline KOOS/HOOS pain subscale, interaction terms between this piece-wise variable and all other separate variables were added, as well as a three-way interaction term between time, treatment and the piece-wise variable. A random intercept was added for individual subjects. The model was designed based on the best fit, Schwartz's Bayesian Information Criterion was 4976.818, and Akaike Corrected Information Criterion was 4968.141. Once the model was constructed for the HOOS/KOOS pain subscale, it was also applied to the other pain outcome measures (VAS-R, VAS-M, m-PDQ).

As a subanalysis, another mixed model for repeated measures was constructed comparing the influence of duloxetine on the development of pain over time for knee and hip OA patients. In this model a fixed variable for joint was added to the above-mentioned model. This way, the difference explained by whether the hip or the knee was the affected joint could be taken into consideration. Further information, as well as the results of this subanalysis, can be found in online supplemental file.

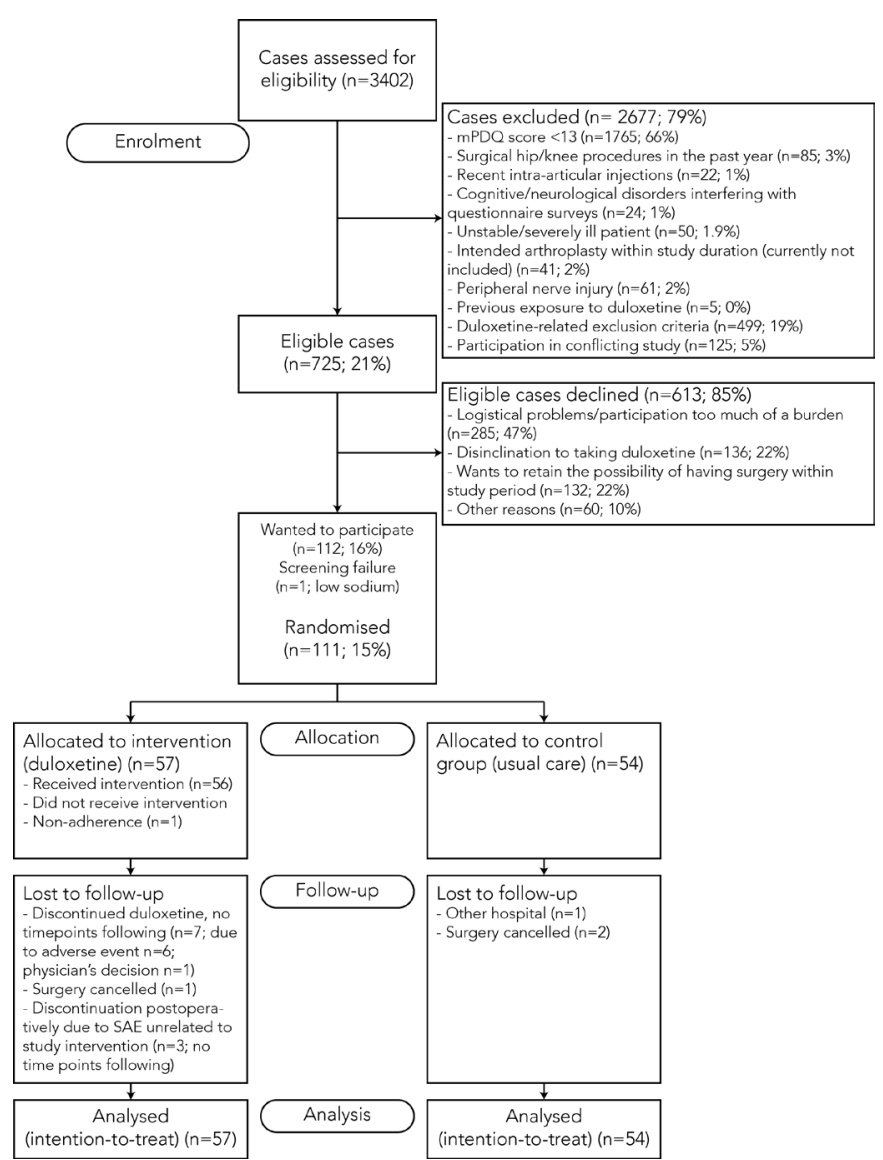

Figure 1 Flow chart of screening and inclusion process. m-PDQ, modified PainDETECT Questionnaire; SAE, serious adverse effect.

\section{RESULTS}

Screening took place over a total number of 3402 patients, $34.1 \%$ of whom had a possible or likely neuropathic pain profile, indicating sensitisation. Of this population, 725 patients were eligible and therefore invited to participate (see figure 1 for the flowchart of the screening and inclusion process). The 112 patients who consented to participate did not differ from non-participants in mean m-PDQ-score $(\mathrm{p}=0.999)$ or hip-versus-knee ratio $(\mathrm{p}=0.184)$. On average, participants were older than nonparticipants (mean difference: 5.2 years; $\mathrm{p}<0.0001$ ) and more often male (38\% males among participants vs $28 \%$ males among non-participants; $\mathrm{p}=0.031$ ).

\section{Non-eligibility and disinclination to participate}

The main reason for declining to participate was the time investment and practical/logistical burden that participation in the study required. Also, disinclination to take duloxetine and having to relinquish the option of another TJA within the 1-year follow-up period were major reasons not to participate in the study.

One patient failed to pass the baseline screening due to a low sodium level, so ultimately 111 patients were included. Baseline characteristics are shown in table 1. Slightly more females (62.2\%) participated and the average participant was 62.7 (SD 8.5) years old. Median 


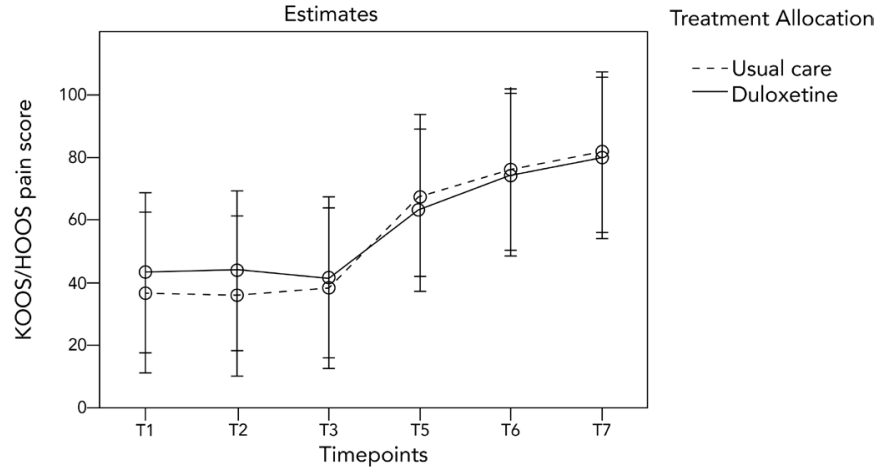

Figure 2 Course of KOOS/HOOS pain subscale per treatment group based on the mixed model for repeated measures using a piece-wise design. HOOS, Hip disability and Osteoarthritis Outcome Score; KOOS, Knee injury and Osteoarthritis Outcome Score.

duration of symptoms was 42 months (IQR 18-72). After randomisation, 57 patients were placed in the intervention group and 54 in the control group. Despite randomisation, there were significant differences in baseline HOOS/KOOS pain subscales $(38.0 \pm 14.0$ in the duloxetine group vs $30.6 \pm 12.7$ in the control group; $\mathrm{p}=0.004$ ) and mean VAS at rest $(46.6 \pm 24.8$ in the duloxetine group vs $58.7 \pm 18.2$ in the control group; $\mathrm{p}=0.004)$. Concurrent back pain was reported by $11.9 \%$ of participants $(7.3 \%$ vs $16.7 \%$ for the intervention vs control group, respectively; $\mathrm{p}=0.151)$. The incidence of other pain conditions (migraine, irritable bowel syndrome, fibromyalgia, chronic neck pain) was below $10 \%$, with no significant differences between the groups.

\section{Postoperative pain}

Figure 2 presents visual report of the course of the KOOS/ HOOS pain subscale over different time points for both groups. The KOOS and HOOS pain subscales showed a skewed distribution 6 months postoperatively. Median score was 86.3 (IQR 64.6-95) in the intervention group and 80.6 (IQR 57.5-92.5) in the control group. Due to the significant difference in KOOS/HOOS pain subscales and VAS pain scales at baseline, the mean change in scores between 6 months postoperatively and baseline was assessed for these measurement outcomes. The mean change in KOOS/HOOS pain subscales was 37.0 (SD 28.1) in the intervention group and 43.3 (SD 26.5) in the control group. At $\mathrm{p}=0.280$, no statistically significant difference was found in change score 6 months post-TJA between the groups (non-parametrically tested).

Based on the multilevel mixed model for repeated measures as described above, table 2 presents the estimated means and differences in pain at different time points between treatment groups.

Table 2 Estimated means $(95 \% \mathrm{Cl})$ and estimated difference $(95 \% \mathrm{Cl})$ based on the mixed model for repeated measures using a piece-wise design

\begin{tabular}{|c|c|c|c|c|c|}
\hline & & Intervention (57) & Control (54) & Difference & Significance \\
\hline \multicolumn{6}{|l|}{ Preoperatively } \\
\hline \multirow{3}{*}{$\begin{array}{l}\text { After } 7 \text { weeks targeted } \\
\text { treatment }\end{array}$} & KOOS/HOOS-p & $44.0(18.3-69.7)$ & $35.7(10.1-61.4)$ & $8.3(1.3-15.3)$ & 0.021 \\
\hline & VAS-R & $42.1(12.1-72.1)$ & $55.2(25.2-85.1)$ & $13.0(4.8-21.2)$ & 0.002 \\
\hline & VAS-M & $55.5(24.5-86.5)$ & 68.8 (37.9-99-8) & $13.3(4.9-21.8)$ & 0.002 \\
\hline \multirow[t]{4}{*}{6 weeks postarthroplasty } & KOOS/HOOS-p & $63.4(37.7-89.1)$ & $67.6(41.9-93.4)$ & $4.3(-3.0-11.5)$ & 0.248 \\
\hline & $\mathrm{mPDQ}$ & $10.7(1.7-19.6)$ & $9.1(0.2-18.1)$ & $1.5(-1.1-4.2)$ & 0.251 \\
\hline & VAS-R & $21.3(-8.7-51.4)$ & $21.8(-8.2-51.8)$ & $0.5(-8.0-8.9)$ & 0.914 \\
\hline & VAS-M & $31.7(0.7-62.7)$ & $25.9(-5.1-56.8)$ & $5.8(-2.8-14.5)$ & 0.187 \\
\hline 6 months postarthroplasty & VAS-M & $25.3(-5.7-56.3)$ & $21.3(-9.7-52.2)$ & $4.0(-4.8-12.8)$ & 0.370 \\
\hline \multirow[t]{4}{*}{12 months postarthroplasty } & KOOS/HOOS-p & $79.8(54.1-105.5)$ & $81.6(55.9-107.3)$ & $1.8(-5.5-9.1)$ & 0.623 \\
\hline & $\mathrm{mPDQ}$ & $4.9(-4.0-13.9)$ & $4.9(-4.0-13.8)$ & $0.1(-2.5-2.6)$ & 0.967 \\
\hline & VAS-R & $12.9(-17.1-43.0)$ & $15.7(-14.3-45.7)$ & $2.8(-5.7-11.3)$ & 0.518 \\
\hline & VAS-M & $19.1(-11.9-50.2)$ & $18.7(-12.2-49.7)$ & $0.4(-8.3-9.1)$ & 0.929 \\
\hline
\end{tabular}

Ranges: KOOS/HOOS pain subscale 0-100; mPDQ -1-38; VAS-R 0-100; VAS-M 0-100.

Bold=primary endpoint, 6 months postarthroplasty.

HOOS, Hip disability and Osteoarthritis Outcome Score; KOOS, Knee injury and Osteoarthritis Outcome Score; m-PDQ, modified PainDETECT Questionnaire; VAS-M, Visual Analogue Scale for pain during Movement; VAS-R, VAS for pain in Rest. 


\section{Results of subanalysis}

The study group consisted of 61 knee OA patients and 50 hip OA patients. The table in online supplemental file presents the estimated means and difference based on the mixed model for repeated measures using a piecewise design including joint as a fixed variable. A significant effect was seen in knee OA patients after 7 weeks of targeted treatment with duloxetine compared with usual care, with an estimated mean KOOS pain subscale score of 47.2 (95\% CI 21.6 to 72.8) for duloxetine and 33.9 (95\% CI 8.3 to 59.5) for usual care (estimated difference $13.3,95 \%$ CI 4.4 to 22.3 ; $\mathrm{p}=0.004$ ). As seen in the table in online supplemental file, the duloxetine treatment does not show a similar effect in hip OA patients, with an estimated mean HOOS pain subscale score of 39.9 (95\% CI 14.0 to 65.7$)$ for duloxetine and 38.0 (95\% CI 12.3 to 63.7 ) for usual care (estimated difference 1.8, $95 \%$ CI -8.0 to $11.7 ; \mathrm{p}=0.714$ ). For both subgroups there was no significant effect of duloxetine treatment on any of the postoperative time points (estimated differences of 4.1 ( $95 \%$ CI -6.1 to $14.3 \mathrm{p}=0.432$ for hip OA patients and estimated differences of 0.5 (95\% CI -9.1 to 10.0 $\mathrm{p}=0.924$ for knee OA patients at 6 months postoperatively. (Online supplemental figure 3 shows the course of the KOOS/HOOS pain subscale for the different treatment groups based on the mixed model for repeated measures including joint groups.

\section{Chronic residual pain}

At 6 months postoperatively, $32.6 \%$ of the intervention group and $31.9 \%$ of the control group scored a KOOS/ HOOS pain subscale $<70$ points, representing moderate chronic residual pain. These percentages decreased to $27.3 \%$ and $31.3 \%$ at 12 months postoperatively for the intervention and control groups, respectively. When looking at hip and knee patients separately, $14.3 \%$ of hip patients and $47.1 \%$ of knee patients had a KOOS/HOOS pain subscale $<70$ sixmonths postarthroplasty. Twelve months postarthroplasty this was $19 \%$ for hip patients and $38 \%$ for knee patients.

\section{Lost to follow-up, protocol violations, adverse effects, and missing data}

Within the intervention group, 12 patients (21.1\%) discontinued duloxetine due to adverse effects (AEs). No subsequent time points were retrieved after these patients' discontinuation, so they were lost to follow-up. Other losses to follow-up constituted approximately $5 \%$ of participants (see figure 1). There were 10 registered protocol violations, nine from another TJA within the follow-up year (two in the intervention group vs seven in the usual care group). These patients all remained in the study up to 1-year follow-up. Three patients from the intervention group discontinued participation during the follow-up period due to serious AEs not related to the intervention. One patient (intervention group) developed post-TKA infection and underwent extensive additional treatment involving surgery and antibiotics.
This patient did remain in the follow-up process up to the end of the study. Another patient (intervention group) suffered from post-TKA aseptic loosening of the tibial component, also remained in the study up to the last follow-up time point, and later on underwent revision surgery. Apart from these discontinuations, some patients did not return their questionnaires for any of the follow-up time points despite phone and/or mail reminders. Complete follow-up of all postoperative time points up to 1 year postoperatively was retrieved in 92 cases $(82.9 \%)$.

\section{DISCUSSION}

In this study, a 7-week preoperative targeted treatment with duloxetine in a study population of end-stage hip/ knee OA patients suffering from sensitisation did not show an effect on postoperative chronic residual pain after THA/TKA. Extensive literature describes the association between signs of sensitisation in OA and chronic residual pain after TJA. ${ }^{1610131416182021}$ Forthcoming was the hypothesis that targeted treatment aimed at desensitisation prior to surgery would reduce chronic residual pain postoperatively. However, the present randomised clinical trial does not support this hypothesis.

Several factors could be playing a role in our findings. First, if we were not successful in identifying the sensitised subpopulation of OA patients, the study population may not have been as sensitised as we anticipated and the treatment effect would be diluted accordingly. However, we used a screening questionnaire specifically modified to measure sensitisation in knee and hip OA patients. Previous studies showed sensitivity and specificity of $50 \%$ and $74 \%$, respectively, for the $>12$ points cut-off (possible sensitisation), whereas a $>18$ points cut-off (likely sensitisation) showed substantially higher sensitivity and specificity (both $80 \%$ ). ${ }^{81}$ It should be noted, however, that these figures are based only on a small study involving knee OA patients and a study performed on a heterogeneous group of patients with low back pain. ${ }^{81}$ We deliberately chose the cut-off point for possible sensitisation, because OA patients are more likely to experience a mixed-pain phenotype with nociceptive and neuropathiclike symptoms due to the multifactorial pathophysiology of OA pain. ${ }^{1}$ A solely neuropathic-like pain experience in $\mathrm{OA}$ is less likely. Moreover, in line with literature we found that $34.1 \%$ of screened OA patients had a possible or likely neuropathic pain profile, thereby increasing the likelihood that we identified the target subpopulation. ${ }^{710-12162338}$

Second, if we were not successful in adequately desensitising patients prior to surgery this could explain the lack of effect on chronic residual pain after TJA. A statistically significant treatment effect of 8.3 points (95\% CI 1.3 to 15.3) was found in the preoperative treatment phase, yet this difference does not seem clinically relevant compared with reported minimally important changes (MIC) of 10 points in literature. ${ }^{39-41}$ It should be noted that these MIC 
values are mostly reported after operative treatments and therefore cannot automatically be extrapolated to relevant changes following conservative treatment. If the effect of desensitisation is too small to make a clinically relevant difference immediately following the treatment phase, this could explain the lack of effect on chronic residual pain after TJA. A detailed analysis of the treatment effect in the preoperative study period was published earlier, ${ }^{42}$ describing more extensively how effects of duloxetine found in previous literature are similar to the effect in this study for knee OA. Still, comparison is only possible to a limited extent due to the more controlled nature of previous studies and investigating only knee OA populations. ${ }^{24-27}$ There is a lack of studies on hip OA patients. Thanks to the enriched nature of this study a greater effect of duloxetine could have been expected when comparing to previous studies. The administered duloxetine regimen was in accordance with the recommended treatment dose based on previous literature, although the treatment duration can be considered relatively short compared with literature. ${ }^{24} 253334$ Future studies could investigate whether a longer preoperative treatment duration would show more effect on chronic residual pain post-TJA. As described in the subanalysis in online supplemental file, the found effect of duloxetine treatment can be principally attributed to the knee OA group of the study population. No effect of duloxetine treatment was found in the hip OA study population. The cause of the lack of effect in the hip OA subgroup of patients can only be speculated on. ${ }^{42}$ Also, for hip OA patients, despite having screened for signs of sensitisation, we found a relatively low percentage of chronic residual pain- $14.3 \%$ after 6 months and 19\% after 12 months-whereas literature reports up to $23 \% .{ }^{21}{ }^{22}$ Consequently, the association between sensitisation in hip OA and development of chronic residual pain after THA is less prominent in this study. The proportion of patients with chronic residual pain is relatively high in knee OA patients after TKA, $47.1 \%$ after 6 months and $38 \%$ after 12 months, compared with up to $34 \%$ in literature. ${ }^{10} 182021$ This was expected due to the enriched nature of our study population. And yet the numbers of the subgroups of knee and hip OA patients are low, rendering generalisability of these findings limited.

Third, it is possible that the effect of duloxetine treatment diminishes after tapering of the treatment dose and that the desensitisation is becoming undone in the (short) interval period between tapering and surgery. This interval period was imposed for safety reasons (see the Methods section). In the previous publication focusing on the preoperative study period, a decrease in treatment effect could be observed after the tapering phase. ${ }^{42}$ This could explain the lack of treatment effect on chronic residual pain. In a recent study, a $30 \mathrm{mg}$ duloxetine regimen was administered 1 day before up to 6 weeks after TKA to knee OA patients with signs of sensitisation; the perioperative duloxetine treatment significantly reduced pain up to 12 weeks postoperatively. ${ }^{27}$ Maybe the treatment timing should have been more suitability and safety of the perioperative period, but no information is reported beyond 12 weeks postoperatively. Studies are needed to determine whether a different timing of preoperative duloxetine treatment continuing up to (or shortly after) TJA has a different effect on chronic residual pain compared with this study.

Fourth, this study centres around the hypothesis that treatment of sensitisation in OA patients curbs development of chronic residual pain after THA/TKA. Although in literature signs of sensitisation are a known predictor for developing chronic residual pain post-TJA, that does not necessarily imply that treatment of the first prevents development of the latter. Our present findings could therefore be in line with a theory by Neogi ${ }^{14}$ : rather than being induced by nociceptive input from the OA pathology, sensitisation should be seen as a trait related to a person's genetic/systemic predisposition to increased pain perception, which is unmasked once nociceptive input is supplied by structural OA pathology. Maybe sensitisation in OA and chronic residual pain post-TJA are both traits of an underlying proneness/vulnerability to enhanced pain experience, which explains why people who develop sensitisation in OA are also at risk of developing chronic residual pain, but treatment of the first does not influence the underlying vulnerability and therefore does not lessen the development of chronic residual pain. As to our knowledge, this is the first study to investigate the direct effect of treatment of sensitisation in OA on chronic residual pain, additional research is needed to reassess the present findings and to further investigate the complex causal pathways in the development of chronic residual pain.

\section{Strengths and limitations}

This study contributes important pragmatic insights to the existing literature. There is an increasing demand for pragmatic studies in the field of OA research. ${ }^{43-45}$ Pragmatic trials attempt to demonstrate whether an intervention works in the reality of daily practice rather than under highly controlled conditions. Pragmatic dimensions of the DOA study are specified in detail in our design study.

There are also limitations to this study. First, the substantial difference in treatment effect of duloxetine in the two different joint groups was not anticipated and somewhat lessens the interpretability of our results for the total study group, as the study population was underpowered to analyse hip and knee OA patients separately. However, by designing a mixed model for repeated measures including joint as a fixed variable (see subanalysis in online supplemental file) we were able to assess the effect of joint group in the study population as a whole. Second, by comparing duloxetine treatment with usual care we can only assess the combination of the pharmacological effect together with the accompanying placebo and nocebo effects. However, to some extent these factors would also play a role in daily administration of this treatment and are therefore relevant for assessing 
the effectiveness of the total intervention. Due to lack of blinding and the high percentage of AEs in the duloxetine treatment group, there is a possibility of a nocebo effect, especially during and shortly after the intervention period. Still, due to the extensive time period between the actual study intervention and the surgery that took place in-between, this effect is not very likely to have influenced the primary endpoint of this study at 6 months post-arthroplasty.

\section{Suitability and safety}

Regarding suitability and safety of duloxetine in the targeted treatment population, the percentage of AEs was high in the intervention group, with $21.1 \%$ of intervention participants discontinuing the study treatment due to AEs. The incidence and nature of the AEs in the treatment period are described in more detail elsewhere. ${ }^{42}$ Also, due to the risk of side effects a substantial proportion of patients was disinclined to participate in the study. This, in combination with the substantial number of contraindications for duloxetine for medical reasons, lessens the practical applicability of duloxetine in general practice for OA patients with accompanying comorbidity.

\section{CONCLUSION}

Based on the results of this study, preoperative targeted treatment with duloxetine in end-stage hip and knee OA patients with sensitisation does not influence postoperative chronic residual pain after arthroplasty. Duloxetine does seem to have a treatment effect on pain in end-stage knee OA patients suffering from sensitisation, but clinically relevant thresholds were not met and applicability seems limited. No treatment effect was found in endstage hip OA patients with sensitisation. The percentage of patients with chronic residual pain in this sensitised study population was relatively high for knee patients (38\%, 12 months post-TKA) but relatively low for hip patients (19\%, 12 months post-THA). Additional studies are needed, especially regarding timing and duration of duloxetine treatment. Other treatment options for OA patients with sensitisation as well as for chronic residual pain should be explored. Dedicated studies specifically addressing these issues in hip OA patients are indicated, considering the apparent differences between hip and knee OA patients found in this study.

\footnotetext{
Author affiliations

${ }^{1}$ Department of Rehabilitation Medicine, University of Groningen, University Medical Center Groningen, Groningen, The Netherlands

${ }^{2}$ Department of Orthopaedic Surgery, University of Groningen, University Medical Center Groningen, Groningen, The Netherlands

${ }^{3}$ Department of Orthopaedic Surgery, Medical Center Leeuwarden, Leeuwarden, The Netherlands

${ }^{4}$ Department of Health Sciences, Community and Occupational Medicine, University of Groningen, University Medical Center Groningen, Groningen, The Netherlands ${ }^{5}$ University of Groningen/UMCG, Groningen, The Netherlands

${ }^{6}$ Department of Orthopaedic Surgery, Martini Hospital Groningen, Groningen, The Netherlands
}

${ }^{7}$ Department of Anaesthesiology, Martini Hospital Groningen, Groningen, The Netherlands

Contributors Conception and design of the study: WR, TB, RS, SB, IvdA-S and MS. Acquisition, analysis and interpretation of data: WR, TB, SB, IvdA-S and MS. Drafting and revision of the manuscript: WR, TB, BD, RS, WZ, TvR, AtH, SB, IvdA-S and MS. Guarantor: WR

Funding This work was supported by the Dutch Arthritis Foundation, Reumafonds (grant number BP 12-3-401).

Competing interests None declared.

Patient consent for publication Not applicable.

Ethics approval The study was approved by the Medical Ethics Committee of University Medical Center Groningen (2014/087).

Provenance and peer review Not commissioned; externally peer reviewed.

Data availability statement Data are available on reasonable request. The deidentified participant database and additional statistical information is available by contacting the corresponding author and reuse is permitted upon reasonable request.

Supplemental material This content has been supplied by the author(s). It has not been vetted by BMJ Publishing Group Limited (BMJ) and may not have been peer-reviewed. Any opinions or recommendations discussed are solely those of the author(s) and are not endorsed by BMJ. BMJ disclaims all liability and responsibility arising from any reliance placed on the content. Where the content includes any translated material, BMJ does not warrant the accuracy and reliability of the translations (including but not limited to local regulations, clinical guidelines, terminology, drug names and drug dosages), and is not responsible for any error and/or omissions arising from translation and adaptation or otherwise.

Open access This is an open access article distributed in accordance with the Creative Commons Attribution Non Commercial (CC BY-NC 4.0) license, which permits others to distribute, remix, adapt, build upon this work non-commercially, and license their derivative works on different terms, provided the original work is properly cited, appropriate credit is given, any changes made indicated, and the use is non-commercial. See: http://creativecommons.org/licenses/by-nc/4.0/.

\section{ORCID iDs}

Wietske Rienstra http://orcid.org/0000-0001-6718-3129

Tim Blikman http://orcid.org/0000-0002-6318-6450

Roy Stewart http://orcid.org/0000-0001-9227-433X

Martin Stevens http://orcid.org/0000-0001-8183-6894

Inge van den Akker-Scheek http://orcid.org/0000-0002-1614-8419

\section{REFERENCES}

1 Neogi T. The epidemiology and impact of pain in osteoarthritis. Osteoarthritis Cartilage 2013;21:1145-53.

2 Hunter DJ, Bierma-Zeinstra S. Osteoarthritis. Lancet 2019;393:1745-59.

3 Kurtz SM, Ong KL, Lau E, et al. Impact of the economic downturn on total joint replacement demand in the United States: updated projections to 2021. J Bone Joint Surg Am 2014;96:624-30.

4 Inacio MCS, Graves SE, Pratt NL, et al. Increase in total joint arthroplasty projected from 2014 to 2046 in Australia: a conservative local model with international implications. Clin Orthop Relat Res 2017;475:2130-7.

5 Kurtz S, Ong K, Lau E, et al. Projections of primary and revision hip and knee arthroplasty in the United States from 2005 to 2030. J Bone Joint Surg Am 2007;89:780-5.

6 Beswick AD, Wylde V, Gooberman-Hill R, et al. What proportion of patients report long-term pain after total hip or knee replacement for osteoarthritis? A systematic review of prospective studies in unselected patients. BMJ Open 2012;2:e000435.

7 Thakur M, Dickenson AH, Baron R. Osteoarthritis pain: nociceptive or neuropathic? Nat Rev Rheumatol 2014;10:374-80.

8 Hochman JR, Davis AM, Elkayam J, et al. Neuropathic pain symptoms on the modified painDETECT correlate with signs of central sensitization in knee osteoarthritis. Osteoarthritis Cartilage 2013;21:1236-42.

9 Ohtori S, Orita S, Yamashita M, et al. Existence of a neuropathic pain component in patients with osteoarthritis of the knee. Yonsei Med $J$ 2012;53:801. 
10 Valdes AM, Suokas AK, Doherty SA, et al. History of knee surgery is associated with higher prevalence of neuropathic pain-like symptoms in patients with severe osteoarthritis of the knee. Semin Arthritis Rheum 2014;43:588-92.

11 Fingleton C, Smart K, Moloney N, et al. Pain sensitization in people with knee osteoarthritis: a systematic review and meta-analysis. Osteoarthritis Cartilage 2015;23:1043-56.

12 Lluch E, Torres R, Nijs J, et al. Evidence for central sensitization in patients with osteoarthritis pain: a systematic literature review. Eur $J$ Pain 2014;18:1367-75.

13 Neogi T, Frey-Law L, Scholz J, et al. Sensitivity and sensitisation in relation to pain severity in knee osteoarthritis: trait or state? Ann Rheum Dis 2015;74:682-8.

14 Neogi T. Structural correlates of pain in osteoarthritis. Clin Exp Rheumatol 2017;35 Suppl 107:75-8.

15 Dimitroulas T, Duarte RV, Behura A, et al. Neuropathic pain in osteoarthritis: a review of pathophysiological mechanisms and implications for treatment. Semin Arthritis Rheum 2014;44:145-54.

16 Arendt-Nielsen L. Pain sensitisation in osteoarthritis. Clin Exp Rheumatol 2017;35 Suppl 107:68-74.

17 Gwilym SE, Keltner JR, Warnaby CE, et al. Psychophysical and functional imaging evidence supporting the presence of central sensitization in a cohort of osteoarthritis patients. Arthritis Care Res 2009;61:1226-34.

18 Skou ST, Graven-Nielsen T, Rasmussen S, et al. Facilitation of pain sensitization in knee osteoarthritis and persistent post-operative pain: a cross-sectional study. Eur J Pain 2014;18:1024-31.

19 Lundblad H, Kreicbergs A, Jansson KA. Prediction of persistent pain after total knee replacement for osteoarthritis. J Bone Joint Surg Br 2008;90:166-71.

20 Wylde V, Palmer S, Learmonth ID, et al. The association between preoperative pain sensitisation and chronic pain after knee replacement: an exploratory study. Osteoarthritis Cartilage 2013;21:1253-6.

21 Wylde V, Sayers A, Lenguerrand E, et al. Preoperative widespread pain sensitization and chronic pain after hip and knee replacement: a cohort analysis. Pain 2015;156:47-54.

22 Wylde V, Hewlett S, Learmonth ID, et al. Persistent pain after joint replacement: prevalence, sensory qualities, and postoperative determinants. Pain 2011;152:566-72.

23 Murphy SL, Phillips K, Williams DA, et al. The role of the central nervous system in osteoarthritis pain and implications for rehabilitation. Curr Rheumatol Rep 2012;14:576-82.

24 Wang G, Bi L, Li X, et al. Efficacy and safety of duloxetine in Chinese patients with chronic pain due to osteoarthritis: a randomized, double-blind, placebo-controlled study. Osteoarthritis Cartilage 2017;25:832-8

25 Wang ZY, Shi SY, Li SJ, et al. Efficacy and safety of duloxetine on osteoarthritis knee pain: a meta-analysis of randomized controlled trials. Pain Med 2015;16:1373-85.

26 Osani MC, Bannuru RR. Efficacy and safety of duloxetine in osteoarthritis: a systematic review and meta-analysis. Korean $J$ Intern Med 2019;34:966-73.

27 Koh IJ, Kim MS, Sohn S, et al. Duloxetine reduces pain and improves quality of recovery following total knee arthroplasty in centrally sensitized patients: a prospective, randomized controlled study. J Bone Joint Surg Am 2019;101:64-73.

28 Blikman T, Rienstra W, van Raaij TM, et al. Duloxetine in osteoarthritis (Doa) study: study protocol of a pragmatic open-label randomised controlled trial assessing the effect of preoperative pain treatment on postoperative outcome after total hip or knee arthroplasty. BMJ Open 2016;6:e010343

29 Rienstra W, Blikman T, Mensink FB, et al. The modified painDETECT questionnaire for patients with hip or knee osteoarthritis: translation into Dutch, cross-cultural adaptation and reliability assessment. PLoS One 2015;10:e0146117.

30 Rienstra W, Blikman T, Dijkstra B, et al. Validity of the Dutch modified painDETECT questionnaire for patients with hip or knee osteoarthritis. Disabil Rehabil 2019;41:941-7.

31 Freynhagen R, Baron R, Gockel U, et al. painDETECT: a new screening questionnaire to identify neuropathic components in patients with back pain. Curr Med Res Opin 2006;22:1911-20.

32 Of H, Information P, Capsules DD. Prescribing information. Cymbalta: Eli Lilly, 2006

33 Chappell AS, Ossanna MJ, Liu-Seifert H, et al. Duloxetine, a centrally acting analgesic, in the treatment of patients with osteoarthritis knee pain: a 13-week, randomized, placebo-controlled trial. Pain 2009;146:253-60.

34 Chappell AS, Desaiah D, Liu-Seifert H, et al. A double-blind, randomized, placebo-controlled study of the efficacy and safety of duloxetine for the treatment of chronic pain due to osteoarthritis of the knee. Pain Pract 2011;11:33-41.

35 Hip disability and Osteoartrhitis outcome score, scoring. HOOS Excel scoring files, 2013. Available: http://www.koos.nu [Accessed Apr 2021].

36 Knee Osteoartrhitis outcome score, scoring. KOOS Excel scoring files, 2012. Available: http://www.koos.nu [Accessed Apr 2021].

37 Roos EM, Lohmander LS. The knee injury and osteoarthritis outcome score (KOOS): from joint injury to osteoarthritis. Health Qual Life Outcomes 2003;1:64.

38 Finan PH, Buenaver LF, Bounds SC, et al. Discordance between pain and radiographic severity in knee osteoarthritis: findings from quantitative sensory testing of central sensitization. Arthritis Rheum 2013;65:363-72.

39 Collins NJ, Prinsen CAC, Christensen R, et al. Knee injury and osteoarthritis outcome score (KOOS): systematic review and meta-analysis of measurement properties. Osteoarthritis Cartilage 2016;24:1317-29.

40 Çelik D, Çoban Özge, Kılıçoğlu Önder. Minimal clinically important difference of commonly used hip-, knee-, foot-, and anklespecific questionnaires: a systematic review. J Clin Epidemiol 2019:113:44-57.

41 Roos EM, Boyle E, Frobell RB, et al. It is good to feel better, but better to feel good: whether a patient finds treatment 'successful' or not depends on the questions researchers ask. Br J Sports Med 2019;53:1474-8.

42 Blikman T. Neuropathic-like symptoms in hip and knee osteoarthritis. University of Groningen, 2020. ISBN: 978-94-6419-011-3. http://hdl. handle.net/(.)6a-997f-0fd5826319ce

43 Zhang W, Moskowitz RW, Nuki G, et al. OARSI recommendations for the management of hip and knee osteoarthritis, part II: OARSI evidence-based, expert consensus guidelines. Osteoarthritis Cartilage 2008;16:137-62.

44 Thorpe KE, Zwarenstein M, Oxman AD, et al. A pragmaticexplanatory continuum indicator summary (Precis): a tool to help trial designers. J Clin Epidemiol 2009;62:464-75.

45 Zwarenstein M, Treweek S, Gagnier JJ, et al. Improving the reporting of pragmatic trials: an extension of the CONSORT statement. BMJ 2008;337:a2390. 